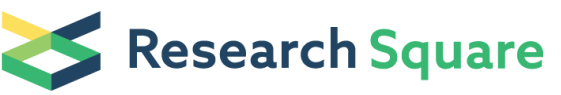 \\ Preprints are preliminary reports that have not undergone peer review. \\ They should not be considered conclusive, used to inform clinical practice, or referenced by the media as validated information.
}

\section{Impairment of the visuospatial working memory in the patients with Parkinson's Disease: an fMRI study}

\section{Shoji Kawashima ( $\nabla$ shoji@med.nagoya-cu.ac.jp )}

Department of Neurology and Neuroscience, Nagoya City University Graduate School of Medical

Science

\section{Yoko Shimizu}

Department of Rehabilitation Medicine, Nagoya City University Graduate School of Medical Science

\section{Yoshino Ueki}

Department of Rehabilitation Medicine, Nagoya City University Graduate School of Medical Science Noriyuki Matsukawa

Department of Neurology and Neuroscience, Nagoya City University Graduate School of Medical Science

\section{Research Article}

Keywords: Mild cognitive impairment, Parkinson's disease, functional MRI, working memory, n-back test

Posted Date: December 17th, 2020

DOI: https://doi.org/10.21203/rs.3.rs-120393/v1

License: (c) (i) This work is licensed under a Creative Commons Attribution 4.0 International License. Read Full License 


\section{Abstract}

Background: Mild cognitive impairment $(\mathrm{MCl})$ is a common symptom in the patients with Parkinson's disease (PD). $\mathrm{MCl}$ is a transitional stage between normal ageing and dementia. The neuropsychological characteristics of the PD-MCl are impairment in frontal executive function and/or visuospatial working memory. The newly modified version of the n-back test, visuospatial n-back test can assess both visuospatial recognition and visuospatial working memory.

Methods: In this study, we aimed to clarify the advantage of visuospatial n-back test as a tool for the detection of the impairment of working memory in the patients with PD. Using functional MRI, we aimed to search the specific brain regions associated with the impairment of visuospatial working memory. The score of 0-back reflects visuospatial recognition, and the scores of 1-back and 2-back reflect visuospatial working memory. We recruited 13 patients with PD-MCl, and 15 patients with cognitive normal PD (PD$\mathrm{CN})$. Group comparisons between PD-MCl and PD-CN were performed for three loads of this test. The correlations between the scores of $n$-back test and task-related activations were analysed for all patients.

Results: We found that the correct answer rate of patients with PD-MCl was lower in the 2-back test than those with PD-CN. However, scores of the 0-back and 1-back tests were not different between the groups. The result of $\mathrm{fMRI}$ showed that the activations within the middle frontal gyrus (MFG) and the inferior parietal lobule (IPL) during the 2-back test were reduced in the patients with PD-MCl.

Conclusions: This study revealed an impaired visuospatial working memory in PD-MCl in association with the reduced activations of MFG and IPL. Combinations of functional neuroimaging and the visuospatial $n$-back test are beneficial to evaluate the impairment of working memory in PD.

\section{Introduction}

Striatal dopamine depletion due to the degeneration of nigrostriatal dopaminergic neurons causes motor disturbances in the patients with Parkinson's disease (PD). The cognitive impairment is common symptoms of PD as well as motor disturbances. The movement disease society (MDS) has published the clinical criteria for dementia in PD (PDD) in 2007 [1], and the MDS Task Force has provided the criteria for mild cognitive impairment (MCI) in PD (PD-MCl) in 2012 [2]. $\mathrm{MCl}$ is a transitional stage between normal ageing and dementia that has been used to detect and treat early dementia [3]. In a recent cohort study, $20 \%$ of de novo patients with PD were classified as PD-MCl at baseline, and $39 \%$ of patients with baseline or incident PD-MCl progressed to dementia during the 5-year follow-up period [4]. The MDS Task Force has advocated two criteria: Level $\mathbb{Q}$ (abbreviated) criteria based on the Montreal Cognitive Assessment (MoCA) score for global cognitive function, and Level II (comprehensive) criteria based on an efficient neuropsychological test battery of subdomains. Although the different criteria produce different classifications of PD-MCl status, both assessment (based on abbreviated and comprehensive criteria), are needed to evaluate cognitive impairment of the PD. 
With regards to cognitive impairment in PD, various studies have revealed that impairments in frontal executive function, including working memory and visual recognition, are especially associated with dementia in PD $[5,6]$. Working memory is responsible for the short-term storage and online manipulation of information necessary for higher cognitive function; impaired working memory can disrupt activities of daily living. One major test to assess working memory is the n-back test, which was developed in the 1950s by Kirchner. Briefly, subjects are presented a sequence of stimuli one-by-one, and they must decide and react immediately if the currently presented stimulus is identical to that presented $\mathrm{N}$ trials ago [7]. Many neuroimaging studies have used the verbal N-back task during functional MRI (fMRI) to explore brain activation associated with working memory processing [8]. Later, a modified version of the visuospatial $n$-back test was developed in fMRI studies to assess visuospatial working memory in normal subjects $[9,10]$. However, the visuospatial $n$-back test has never been used in the neuroimaging study for patients with PD.

This study aimed to investigate fMRI and neuropsychological findings using the visuospatial version of the n-back test in patients with PD-MCl and cognitively normal PD. We hypothesised that the patients with $\mathrm{PD}-\mathrm{MCl}$ show decreased brain activation in association with impaired visuospatial working memory.

\section{Methods}

\section{Participants}

All subjects were recruited from the Department of Neurology at Nagoya City University Hospital. We enrolled 28 right-handed patients with PD (18 males, 10 females) and 12 age-matched normal subjects in the study. All patients included in the study fulfilled the United Kingdom Parkinson's Disease Brain Bank Criteria for clinical diagnosis, and none had a disease other than PD that affected motor and cognitive function. The patients were assessed with the Unified Parkinson's Disease Rating Scale (UPDRS) and the Hoehn and Yahr Scale at the time of inclusion in the study. The patients were excluded if they had dementia according to the criteria for PD dementia provided by the Movement Disorder Society Task Force [1]. We excluded also patients if they had depression, severe insomnia, severe hearing loss, or any other disease that might severely interfere with the fMRI. The study was approved by the local Ethical Committee and complied with national legislation and the Declaration of Helsinki guidelines. All the subjects provided written informed consent prior to the data acquisition.

PD-MCI was classified according to the criteria provided by the Movement Disorder Society Task Force. In Level II criteria, the detection thresholds of -2 SD had significant impact on the discriminative validity of all measures in the MDS neuropsychological battery [12]. In this study, PD-MCI was defined when patients' scores were 2 SD below the normative mean score of the neuropsychological assessments, and it was defined when their impairment on at least two tests represented by either two impaired tests in one cognitive domain (single domain impairment) or one impaired test in two different cognitive domains (multi domain impairment) [2]. The patients were classified as PD-CN when scores were within 2 SD of the normative mean score. 


\section{Neuropsychological test}

Movement disorder specialists performed the complete neuropsychological battery in all the patients and the normal subjects. The MMSE and MoCA were used to assess global cognitive impairment.

Psychomotor speed and attention of subjects were tested with Trail-Making Test Part A (TMT-A) and the Paced Auditory Serial Addition Test (PASAT). Attention and rapid set shifting were tested with TrailMaking Test Part B (TMT-B). Verbal memory was tested using the delayed recall of the Auditory Verbal Learning Test (AVLT); language was tested with the Verbal Fluency Test (total number of words in 60 seconds), and visuospatial function was tested using the visuospatial version of 0-back test. Visuospatial working memory is accessed using visuo-spatial 1-back and 2-back test.

\section{Visuospatial n-back test}

The test used in this study is a modified version of visuospatial n-back test which was reported in fMRI studies for normal subjects $[9,10]$. The patients were asked to perform the tests with 3 load levels during the fMRI. The stimuli were white squares randomly presented in 1 of 8 spatial locations on a screen, through a mirror positioned on a head-coil. The presentation of the stimuli was controlled by a program (Presentation software) that initiated the acquisition of the MRI and the behavioural data. For the 0-back test, the subjects were instructed to press the left button with their index finger when a white square was presented. For the 1-back test, the patients pressed the left button whenever a stimulus was presented in the same location as the previous stimulus. For the 2-back test, when the stimulus was presented in any other location, the patients were instructed to press the right button with the middle finger. The patients were instructed to press the right button when the stimulus appeared in any other location. The higher the number $\mathrm{n}$ requires the higher level of attention and working-memory.

\section{Imaging protocol}

To detect brain regions activated in the subjects performing the visuospatial n-back test, we used the block-design $\mathrm{fMRI}$, which alternated between n-back test and rest conditions. For n-back test conditions, the white square was randomly presented for 2 second in 1 of 8 possible locations on screen; a black screen was presented for 1 second after stimulus presentation. Each test condition consisted of 15 trials over the course of 45 second; each rest condition lasted for 15 second. During a single scan, each test condition was repeated 4 times, in numerical order (0-1-2 back). Thus, each condition included 60 trials (Fig 1).

\section{Fig 1. Experimental protocol}

The figure shows the protocol of n-back task used in this study. To detect the activated brain regions associated with the visuospatial n-back task, we used a blocked design fMRI, alternating n-back test conditions and rest conditions. In n-back task conditions, the white square was presented for $2 \mathrm{~s}$ at random in 1 of 8 possible locations on screen, and black screen was presented for $1 \mathrm{~s}$ after the presentation of stimuli. In the 0 -back test, the subjects were instructed to press the left button with their 
index finger when the white square was presented in predetermined locations. In the 1-back test, the subjects were instructed to press the left button when the stimulus presented in the same location as the previous one. In the 2-back test, the subjects were instructed to press the right button with middle finger when the stimulus presented in any other location. When in any other location, the subjects were instructed to press right button.

\section{Image data acquisition and analysis}

All MRI were acquired with a Siemens Skyra syngo MR E11 3.0 T scanner (Siemens, Germany). Highresolution T1-weighted images were acquired via volumetric 3D spoiled gradient recall sequence. Acquisition parameters were as follows: repetition time $(T R)=1900 \mathrm{~ms}$, echo time $(T E)=2.43 \mathrm{~ms}$, flip angle $(F A)=9$, field of view $(F O V)=256 \times 256 \mathrm{~mm}$, slice thickness $=1 \mathrm{~mm}$, slice gap $=0$, voxel size $=1 \times$ $1 \times 1 \mathrm{~mm}$, number of slices $=176$. The fMRI measurements were performed using a gradient echo EPI sequence: repetition time $(T R)=2500 \mathrm{~ms}$, echo time $(T E)=30 \mathrm{~ms}$, slice thickness $=3 \mathrm{~mm}$, total 149 volumes, with matrix size of $64 \times 64$ and field of view of $192 \times 192 \mathrm{~mm}$, resulting in voxel size of $3 \times 3 \times 3$ $\mathrm{mm}$.

All the images were pre-processed and analysed with Matlab (version 8.1, The Mathworks Inc, Natick, MA) and SPM8 software (Department of Cognitive Neurology, London). Images were realigned to correct for movement and normalised to Montreal Neurologic Institute (MNI) space. The transformed image data were smoothed with a Gaussian philtre (full width at half-maximum $=10 \mathrm{~mm}$ ).

The image data were analysed with a random effect procedure and a parametric model to identify the brain areas where the activation correlated with the task. Group comparisons between PD-MCI and PD-CN were performed for 3 loads of the n-back test, the 1-back vs. 0-back condition and the 2-back vs. 0-back condition. The correlations between score on the n-back test and task-related activation were analysed for all the patients. The statistical significance was established at $\mathrm{P}<0.01$ (uncorrected) with cluster size $>50$ voxels in group analysis, and $\mathrm{P}<0.001$ (uncorrected) with cluster size $>50$ in correlation analysis.

\section{Statistical analysis}

The group differences in the clinical profiles were analysed by the t-test as appropriate. The scores on $\mathrm{n}$ back tests and reaction times were analysed using one-way analysis of variance (ANOVA), because each load level required several neuropsychological cognitive domains. SPSS version 15.0 was used for these analyses. $P<0.05$ was considered significant.

\section{Results}

\section{Clinical profiles of patients with PD-MCl and PD-CN}

The demographic and clinical profiles of patients with PD are summarised in Table 1. A total of 13 patients were classified as $\mathrm{MCl}(\mathrm{PD}-\mathrm{MCl}) ; 15$ patients were classified as cognitively normal (PD-CN). The UPDRS motor scores of the patients with PD-MCl were significantly higher than those of the patients with 
PD-CN $(F(26)=13.542, P<0.001)$. Age, Hoehn and Yahr stage, and disease duration did not differ between groups (Age: $F(26)=1.922, P=0.177$, Hoehn and Yahr stage: $F(26)=0.039, p=0.844$, disease duration : $F(26)=0.097, P=0.758)$. There were no differences among the groups in daily doses of LDOPA $(F(26)=0.669, P=0.421)$, total LDOPA and LDOPA equivalent daily dose of dopamine agonist (LEDD) $(F(26)=0.018, P=0.895)$. In the comparisons of global cognition, there were no group difference in MMSE scores $(F(26)=2.992, P=0.096)$; however, the MoCA scores were significantly lower in the patients with PD$\mathrm{MCl}$ in those with PD-CN $(\mathrm{F}(26)=8.879, \mathrm{P}=0.006)$. In comparisons of neuropsychological subdomains, scores on the PASAT, AVLT, and Verbal Fluency Test were lower in the patients with PD-MCI in those with PD-CN (PASAT: $F(26)=6.906, P=0.014$, AVLT: $F(26)=10.381, P=0.003$, Verbal Fluency Test: $F(26)=8.469$, $P=0.007)$. Patients with PD-MCl took a significantly longer time to complete the TMT-A and TMT-B (TMTA: $F(26)=12.243, P=0.002$, TMT-B: $F(26)=28.155, P<0.001)$.

Table 1. Baseline characteristics of the patients with PD-MCI and PD-CN PD-MCI PD-CN
$\mathrm{n}=13$
$\mathrm{n}=15$
$P$ value

\begin{tabular}{llll}
\hline Age & $69.4 \pm 2.8$ & $67.3 \pm 4.6$ & N.S. \\
\hline Men & 8 & 10 & N.S. \\
\hline Disease Duration (year) & $5.0 \pm 3.2$ & $5.4 \pm 3.5$ & N.S. \\
\hline HY stage & $2.1 \pm 0.8$ & $2.1 \pm 0.7$ & N.S. \\
\hline UPDRS part3 & $19.8 \pm 8.6$ & $10.1 \pm 5.2$ & P $<0.01$ \\
\hline LDOPA (mg) & $262 \pm 153$ & $223 \pm 90$ & N.S. \\
\hline LEDD (mg) & $296 \pm 199$ & $287 \pm 151$ & N.S. \\
\hline MMSE & $26.9 \pm 1.8$ & $28.1 \pm 1.9$ & N.S. \\
\hline MoCA & $21.1 \pm 3.0$ & $24.3 \pm 2.8$ & P $<0.01$ \\
\hline TMT-A (second) & $65.6 \pm 21.0$ & $43.8 \pm 11.2$ & P $<0.01$ \\
\hline TMT-B (second) & $276 \pm 107.5$ & $111 \pm 50.4$ & P $<0.001$ \\
\hline PASAT & $21.2 \pm 6.1$ & $27.2 \pm 6.2$ & P $<0.05$ \\
\hline AVLT (delayed recall) & $5.5 \pm 3.2$ & $9.1 \pm 2.9$ & P $<0.01$ \\
\hline Verbal fluency (words) & $12 \pm 2.9$ & $15.5 \pm 3.1$ & P $<0.01$
\end{tabular}

Duration: Disease duration; HY stage: Hoehn and Yahr stage; UPDRS motor: motor sections of united PD rating scale; LEDD: L-dopa equivalent daily dose of dopamine agonist. Calculation of LDDD for each patient was based on theoretical equivalence to L-dopa as follows: L-dopa dose + L-dopa dose $\times 1 / 3$ [if on entacapone + bromocriptine $(\mathrm{mg}) \times 10+$ cabergoline or pramipexole $(\mathrm{mg}) \times 67+$ ropinirole $(\mathrm{mg}) \times 20+$ pergolide $(\mathrm{mg}) \times 100+$ 
apomorphine $(\mathrm{mg}) \times 8$ ]. MoCA, Montreal Cognitive Assessment, PASAT, Paced Auditory Serial Addition Test; AVLT, Delayed recall of Auditory Visual Learning Test; N.S., not significant

\section{Visuospatial n-back test}

The reaction times and correct answer rates for all 3 tests are summarised in Fig 2. The reaction times of all $n$-back tests were significantly longer in the patients with PD-MCl, compared with the PD-CN (0-back: $F(26)=7.873, P=0.009,1$-back: $F(26)=4.348, P=0.047,2$-back: $F(26)=4.792, P=0.038)$. In the correct answer rate, there was a significant difference in the score of 2-back test $(F(26)=6.001, P=0.021)$. Scores on the 0 -back and 1-back tests were not different between the groups $(0$-back: $F(26)=2.519, P=0.121$; 1 back: $F(26)=3.013, P=0.094)$.

\section{Fig 2. Performance of the visuospatial n-back test}

1. The figure shows rates of correct answers for the patients with PD-CN and PD-MCl. The scores on the 0-back test did not differ significantly between groups. The scores on the 2-back test of PD-MCl were significantly lower than that of PD-CN.

2. The figure shows the response time to press correct button. The patients with PD-MCl took significantly longer time to respond. * $P<0.05, * \star P<0.01$

\section{Correlation between scores on the n-back test and other factors}

The correlations between scores on the n-back test and other factors are presented in Table 2. There was a significant negative correlation between the scores on the 1-back test and the score on UPDRS Part 3 $(R=-0.41, P<0.05)$ and between the scores on the 2-back test and the score on UPDRS Part $3(R=-0.42$, $P<0.05)$. Additionally, we found positive correlations between scores on MoCA and the 1-back test $(R=0.39, P<0.05)$, as well as between scores on MoCA and the 2-back test $(R=0.41, P<0.05)$. We also found a negative correlation between scores on the TMT-B and the 0 -back test $(R=-0.50, P<0.01)$, however, there was no correlation between scores on TMT-A and any load of the n-back test.

\section{Table 2. Correlations between scores on the n-back test and other factors}




\begin{tabular}{|c|c|c|c|c|c|c|}
\hline & & 0-back & 1-back & 2-back & PASAT & TMT-B \\
\hline \multirow[t]{2}{*}{ MoCA } & Peason' R & & 0.39 & 0.413 & 0.35 & -0.54 \\
\hline & $\mathrm{p}$ value & N.S. & 0.040 & 0.029 & 0.041 & 0.003 \\
\hline \multirow[t]{2}{*}{ TMT-A } & Peason' R & & & & & 0.524 \\
\hline & $\mathrm{p}$ value & N.S. & N.S. & N.S. & N.S. & 0.004 \\
\hline \multirow[t]{2}{*}{ TMT-B } & Peason' R & -0.495 & -0.5 & & -0.5 & 1 \\
\hline & $\mathrm{p}$ value & 0.007 & 0.007 & N.S. & 0.007 & \\
\hline \multirow[t]{2}{*}{ UPDRS part3 } & Peason' R & & -0.406 & -0.415 & -0.406 & 0.685 \\
\hline & $\mathrm{p}$ value & N.S. & 0.032 & 0.028 & 0.032 & 0.001 \\
\hline
\end{tabular}

N.S.: not significant

\section{fMRI}

The fMRI group analyses were performed to compare the patients with PD-MCl and the patients with PD$\mathrm{CN}$. MNI coordinates of the centre of activation and cluster size for significant findings are summarised in Table 3. For the 0-back test, the PD-MCl group presented significantly reduced brain activation within the right inferior frontal gyrus, left superior frontal gyrus and left medial frontal gyrus. For the 1-back test, significant differences between the groups were observed for right IFG, bilateral superior parietal lobule and bilateral cuneus. For the 2-back test, the PD-MCl group presented significantly reduced brain activation within right inferior parietal lobule (IPL), right middle frontal gyrus (MFG), left superior parietal lobule, left lateral globus pallidus, left cerebellar tonsil and left precentral gyrus. For the 2-back versus 0back condition, the patients with PD-MCl presented reduced activation within the bilateral MFG and IPL, compared to the patients with PD-CN. The images of significant voxels for each load of the n-back test are presented in Fig 3.

Table 3. MNI coordinates of the centre of activation for each load of the $\mathrm{n}$-back test 
0-back test

\begin{tabular}{lllllll} 
Inferior Frontal Gyrus & $\mathrm{R}$ & 395 & 32 & 16 & -18 & 3.96 \\
\hline Superior Frontal Gyrus & $\mathrm{L}$ & 357 & -24 & 40 & 44 & 4.13 \\
\hline Medial Frontal Gyrus & $\mathrm{L}$ & 250 & -8 & 48 & 10 & 3.45
\end{tabular}

\section{1-back test}

\begin{tabular}{lllllll} 
Inferior Frontal Gyrus & $\mathrm{R}$ & 847 & 28 & 20 & -22 & 3.99 \\
\hline Superior Parietal Lobule & $\mathrm{R}$ & 820 & 30 & -52 & 56 & 3.98 \\
\hline Inferior Semi-Lunar Lobule & $\mathrm{L}$ & 714 & -18 & -64 & -36 & 4.14 \\
Cuneus & $\mathrm{R}$ & 654 & 14 & -82 & 26 & 4.11 \\
\hline Middle Occipital Gyrus & $\mathrm{R}$ & 543 & 44 & -82 & 16 & 3.78 \\
\hline Superior Temporal Gyrus & $\mathrm{L}$ & 487 & -44 & -40 & 10 & 4.30 \\
Cuneus & $\mathrm{L}$ & 422 & -12 & -86 & 28 & 4.00 \\
Fusiform Gyrus & $\mathrm{R}$ & 353 & 42 & -44 & -16 & 3.84 \\
\hline Superior Parietal Lobule & $\mathrm{L}$ & 342 & -30 & -50 & 52 & 3.32 \\
\hline
\end{tabular}

\section{2-back test}

\begin{tabular}{lllllll}
\hline Inferior Parietal Lobule & $\mathrm{R}$ & 2008 & 40 & -48 & 46 & 4.72 \\
Middle Frontal Gyrus & $\mathrm{R}$ & 1602 & 44 & 24 & 36 & 4.49 \\
Middle Frontal Gyrus & $\mathrm{R}$ & 753 & 38 & 46 & -14 & 4.32 \\
\hline Superior Parietal Lobule & $\mathrm{L}$ & 752 & -28 & -50 & 46 & 3.91 \\
\hline Lateral Globus Pallidus & $\mathrm{L}$ & 643 & -24 & -8 & 2 & 3.92 \\
\hline Cerebellar Tonsil & $\mathrm{L}$ & 457 & -30 & -62 & -38 & 3.12 \\
Precentral Gyrus & $\mathrm{L}$ & 436 & -62 & 14 & 8 & 4.78
\end{tabular}

\section{2-back test-0-back test}

\begin{tabular}{lllllll}
\hline Inferior Parietal Lobule & $\mathrm{R}$ & 1031 & 52 & -36 & 40 & 3.74 \\
\hline Middle Frontal Gyrus & $\mathrm{L}$ & 810 & -32 & 22 & 32 & 4.27 \\
\hline Middle Frontal Gyrus & $\mathrm{R}$ & 621 & 28 & 16 & 56 & 3.85 \\
\hline Middle Frontal Gyrus & $\mathrm{R}$ & 386 & 42 & 22 & 40 & 3.80
\end{tabular}




\begin{tabular}{lllllll} 
Inferior Parietal Lobule & $\mathrm{L}$ & 320 & -36 & -54 & 38 & 3.49 \\
\hline Middle Occipital Gyrus & $\mathrm{R}$ & 312 & 26 & -72 & 6 & 3.91 \\
\hline
\end{tabular}

Coordinates $\mathrm{x}, \mathrm{y}$ and $\mathrm{z}$ refer to the anatomical location of the Montreal Neurological Institute space for local maxima of clusters.

\section{Fig 3. fMRI analyses comparing PD-MCI and PD-CN}

The figure shows the results for the 0-back test (A), 1-back test (B) and 2-back test (C). The coloured regions indicate significantly lower brain activation in $\mathrm{PD}-\mathrm{MCl}$, as compared with PD-CN. All the images presented at $\mathrm{P}<0.01$ (uncorrected) with cluster size $>50$ voxels in analysis.

The correlations between the scores on the n-back test and task-related activation are shown in Fig 4 . We found a positive correlation between the score on the 2-back test within the right IPL and the scores on the 1-back test within superior parietal lobule and MFG.

\section{Fig 4. Correlation analyses}

a. The image shows the correlation between the scores on the 1-back test and activation of the 1-back test.

b. The image shows the correlation between the scores on the 2-back test and activation of the 2-back test.

\section{Discussion}

This study aimed to investigate the visuospatial working memory and fMRI findings of the patients with PD-MCl and PD-CN, using the visuospatial n-back test. The scores on the 0-back and 1-back test did not differ significantly between groups. By contrast, the scores of the 2-back test in the patients with PD-MCl was significantly lower than PD-NC. It suggested more severe impairment of the visuospatial working memory in the patients with PD-MCl. The neuroimaging findings associated with visuospatial working memory showed that the patients with PD-MCl had functional deactivation within bilateral MFG and IPL.

Concerning PD-MCl, a meta-analysis reported the prevalence of PD-MCl as $26 \%$ [13], and another reported as $55 \%$ among patients with mean disease duration > 10 years [14]. In further, approximately $10 \%$ of patients with PD convert from $\mathrm{MCl}$ to dementia each year [15]. Therefore, the diagnosis of PD-MCI and cognitive assessment including working memory are important for clinical management. The cognitive impairment in PD is caused by complex pathology. Diffuse Lewy bodies [16-18], Alzheimer disease [17, 18], loss of cholinergic neurones [19], loss of medial nigral dopaminergic neurones [20], and serotonergic and noradrenergic deficits [21] are implicated in dementia in PD. 
Recently, various neuroimaging studies are focusing on PD-MCl to examine which type of pathology is a dominant cause of cognitive impairment. Some studies using quantitative susceptibility mapping have indicated that brain iron deposition is linked with cognitive severity in PD [22, 23]. Thomas et al. reported that whole brain regression analyses of PD identified QSM increases that covaried with lower MoCA scores in the hippocampus and thalamus, with poorer visual function, and with higher dementia risk scores in the parietal, frontal, and medial occipital cortices. To search the brain regions associated with cognitive function in patients with PD, the MoCA has mostly been used to test covariates in recent neuroimaging research. In the present study, we found a significant correlation between MoCA scores and 2-back test scores, indicating that the visuospatial 2-back test is suitable for the neuroimaging analysis of fMRI. Our results revealed that the 2-back test correlated positively with the activation of the right IPL, and that the 1-back test correlated with the superior parietal lobule and MFG. Furthermore, subtracting the activated regions in the 0 -back test from those of the 2-back test showed that functional activation in the bilateral MFG and IPL was lower in PD-MCI than in PD-CN. The activated regions of the 2-back test are associated with visuospatial recognition, motor function, and visuospatial working memory. The activated regions of the 0 -back test are associated with visuospatial recognition and motor function. Therefore, subtracting the activated regions in the 0-back test from those in the 2-back test is associated with visuospatial working memory. Based on these results, the deactivation within MFG and IPL are associated with the impairment of visuospatial working memory in the patients with PD-MCl.

Herein, we discuss about the deactivation of the MFG and IPL. First, concerning the IPL, one post-mortem autopsy report demonstrated that patients with PDD had reduced levels of dopaminergic transporter in the caudate, precuneus, and IPL [21]. Another study that used connectivity analysis of resting-state fMRI to target newly diagnosed PD revealed that patients with PD-MCl showed decreased functional connectivity between the posterior cingulate cortex and posterior IPL [24]. Second, concerning the MFG, $\mathrm{Xu}$ et al. reported that the fronto-striatal functional connectivity degree in the right globus pallidum was negatively correlated with that in the left MFG and disease duration [25]. Based on these reports, reduced activation within MFG and IPL in the present study may have been caused by disease-associated dysfunction of the cortico-striatal neural network in PD.

Various studies report conflicting results of the fMRI during tasks that require working memory. A recent fMRI study that incorporated the n-back task reported that functional activity of de novo patients with PD, compared with controls, was increased in right dorso-lateral prefrontal cortex (including MFG) [26]. The results suggest compensation to maintain behavioural performance in the presence of de novo network deficits. In contrast, Simioni et al. reported that patients with PD who were off dopamine replacement therapy displayed reduced activation in prefrontal and bilateral parietal cortex, compared with controls [27]. L-DOPA seems to both boost engagement of a task-specific prefrontal region and strengthen a putative compensatory caudate-cortical network of the patients with PD [27]. Although the discrepancy in the results of neuroimaging studies may depend on the patient sample and protocol used, the pathophysiology of working memory impairment in PD may depend on the extent of compensation for network dysfunction. 
This study had some limitations. First, performance of neuropsychological tests for executive function is influenced by several factors. Disease-associated impairment of working memory, as well as psychosis, depression and daytime sleepiness, may affect performance. The visuospatial n-back test, in particular, requires continuous attention. Increased variability in subjects' performance on the 2-back test, compared with the 1-back test, results in decreased statistical power. Second, although the LDOPA and the LEDD doses were similar between groups, we cannot rule out the possibility that variety of the dopaminergic agonist may have had differing impacts on performance. Third, because the present study had a relatively small sample size, the statistical power to analyze the neuropsychological data of the n-back test and to search for significant voxels in the fMRI analysis was limited.

However, the protocol of this study has two strengths. First, the visuospatial n-back test can assess both visuospatial recognition and working memory in a single test with three different loads. Second, the subjects pushed a button for each stimulus, so we could also analyze reaction time. The patients with PD-MCl took a significantly longer time than those with PD-CN to react all three loads of the N-back test. These advantages allowed us to search specific regions associated with visuospatial working memory by subtracting of activated region of the 0-back test from that in the 2-back test. The observed findings are more regional than those typically presented for normal individuals. The dysfunction of MFG and IPL may predict cognitive impairment in PD.

\section{Conclusions}

This study revealed an impaired visuospatial working memory in PD-MCl in association with the reduced activations of MFG and IPL. Combinations of functional neuroimaging and the visuospatial n-back test are beneficial to evaluate the impairment of working memory in PD.

\section{Abbreviations}

fMRI, functional magnetic resonance imaging; PD, Parkinson disease; PD-CN, PD with cognitive normal; PD-MCl, PD with mild cognitive impairment; MoCA, Montreal Cognitive Assessment; TMT-A, Trail-Making Test Part A; TMT-B, Trail-Making Test Part B; PASAT, Paced Auditory Serial Addition Test; AVLT, Auditory Verbal Learning Test; IPL, inferior parietal lobule; MFG, middle frontal gyrus

\section{Declarations}

\section{Acknowledgements}

We wish to thank Dr. Satoshi Tanaka for technical support of functional MRI analysis. We are also grateful to the patients who took part in this study.

\section{Authors' contributions}


SK: drafting the manuscript, writing; study concept and design; acquisition and interpretation of data. YS: study concept and design; acquisition of data. YU: study concept and design; review and critique. NM: study concept and design; review. All authors have approved the manuscript.

\section{Funding}

This study was supported in part by Grants-in-Aid for Scientific Research on Priority Areas (Grant number is $15 \mathrm{~K} 16362)$.

\section{Availability of data and materials}

The datasets used in this study are available from the corresponding author on reasonable request.

\section{Ethics approval and consent to participate}

This study was approved by the Institutional Review Board of Nagoya City University Hospitals. All subjects gave written informed consent. This study was complied with national legislation and the Declaration of Helsinki guidelines.

\section{Consent for publication}

Not applicable.

\section{Competing interests}

The authors declare that they have no competing interests.

\section{References}

1. Emre M, Aarsland D, Brown R, Burn D, Duyckaerts $C$, Mizuno Y, et al. Clinical diagnostic criteria for dementia associated with Parkinson's disease. Mov Disord. 2007;22(12):1689-707; quiz 1837.

2. Litvan I, Goldman JG, Troster Al, Schmand BA, Weintraub D, Petersen RC, et al. Diagnostic criteria for mild cognitive impairment in Parkinson's disease. Movement Disorder Society Task Force guidelines. Mov Disord. 2012;27(3):349-56.

3. Petersen RC, Roberts RO, Knopman DS, Boeve BF, Geda YE, Ivnik RJ, et al. Mild cognitive impairment: ten years later. Arch Neurol. 2009;66(12):1447-55.

4. Pedersen KF, Larsen JP, Tysnes OB, Alves G. Natural course of mild cognitive impairment in Parkinson disease: A 5-year population-based study. Neurology. 2017;88(8):767-774.

5. Lee JE, Cho KH, Song SK, Kim HJ, Lee HS, Sohn YH et al. Exploratory analysis of neuropsychological and neuroanatomical correlates of progressive mild cognitive impairment in Parkinson's disease. JNNP. 2013;85(1):7-16.

6. Williams-Gray $\mathrm{CH}$, Evans JR, Goris A, Foltynie T, Ban M, Robbins TW et al. The distinct cognitive syndromes of Parkinson's disease: 5 year follow-up of the CamPaIGN cohort. Brain. 2009;132(Pt 
11):2958-69.

7. Kirchner WK. Age differences in short-term retention of rapidly changing information. $J$ Exp Psychol. 1958;55(4):352-8.

8. Bradley VA, Welch JL, Dick DJ. Visuospatial working memory in Parkinson's disease. JNNP. 1989;52(11):1228-35.

9. Cohen JD, Perlstein WM, Braver TS, Nystrom LE, Noll DC, Jonides J et al. Temporal dynamics of brain activation during a working memory task. Nature. 1997;386(6625):604-8.

10. Courtney SM, Petit L, Maisog JM, Ungerleider LG, Haxby JV. An area specialized for spatial working memory in human frontal cortex. Science. 1998;279(5355):1347-51.

11. Carlson S, Martinkauppi S, Rama P, Salli E, korvenoja A, Aronen HJ. Distribution of cortical activation during visuospatial $n$-back tasks as revealed by functional magnetic resonance imaging. Cereb Cortex. 1998;8(8):743-52.

12. Bezdicek O, Nicolai T, Michalec J, Ruzicka F, Havrankova P, Roth J, et al. The Diagnostic Accuracy of Parkinson's Disease Mild Cognitive Impairment Battery Using the Movement Disorder Society Task Force Criteria. Mov Disord Clin Pract. 2016;8;4(2):237-244.

13. Aarsland D, Bronnick K, Williams-Gray C, Weinraub D, Marder K, Kulisevsky J, et al. Mild cognitive impairment in Parkinson disease: a multicenter pooled analysis. Neurology. 2010; 75(12):1062-9.

14. Janvin C, Aarsland D, Larsen JP, Hugdahl K. Neuropsychological profile of patients with Parkinson's disease without dementia. Dement Geriatr Cogn Disord. 2003;15(3):126-31.

15. Aarsland D, Andersen K, Larsen JP, Lolk A, Nielsen H, Kragh-Sorensen P. Risk of dementia in Parkinson's disease: a community-based, prospective study. Neurology. 2001;56(6):730-6.

16. Harding AJ, Halliday GM. Cortical Lewy body pathology in the diagnosis of dementia. Acta Neuropathol. 2001;102(4):355-63.

17. Mattila, PM, Roytta M, Torikka H, Dickson DW, Rinne JO. Cortical Lewy bodies and Alzheimer-type changes in patients with Parkinson's disease. Acta Neuropathol. 1998;95(6): 576-82.

18. Irwin DJ, White MT, Toledo JB, Xie SX, Robinson JL, Van Deerlin V, et al. Neuropathologic substrates of Parkinson disease dementia. Ann Neurol. 2012;72(4):587-98.

19. Tiraboschi P, Hansen LA, Alford M, Sabbagh MN, Schoos B, Masliah E, et al. Cholinergic dysfunction in diseases with Lewy bodies. Neurology. 2000;54(2):407-11.

20. Rinne JO, Rummukainen J, Paljarvi L, Rinne UK. Dementia in Parkinson's disease is related to neuronal loss in the medial substantia nigra. Ann Neurol. 1989;26(1):47-50.

21. Buddhala C, Loftin SK, Kuley BM, Cairns NJ, Campbell MC, Perlmutter JS. Dopaminergic, serotonergic, and noradrenergic deficits in Parkinson disease. Ann Clin Transl Neurol. 2015; 2(10):949-59.

22. Thomas GEC, Leyland LA, Schrag AE, Lees AJ, Acosta-Cabronero J, Weil RS. Brain iron deposition is linked with cognitive severity in Parkinson's disease. JNNP. 2020;91(4):418-425. 
23. Uchida Y, Kan H, Sakurai K, Arai N, Daisuke K, Shoji K, et al. Voxel-based quantitative susceptibility mapping in Parkinson's disease with mild cognitive impairment. Mov Disord. 2019;34:1164-1173.

24. Hou Y, Yang J, Luo C, Song W, Ou R, Liu W. Dysfunction of the Default Mode Network in Drug-Naive Parkinson's Disease with Mild Cognitive Impairments: A Resting-State fMRI Study. Front Aging Neurosci. 2016;8:247.

25. Xu J, Zhang J, Wang J, Li G, Hu Q, Zhang Y. Abnormal fronto-striatal functional connectivity in Parkinson's disease. Neurosci Lett. 2016;613:66-71.

26. Trujillo JP, Gerrits NJ, Vwltman DJ, Berendse HW, van der Werf YD, van den Heuvel OA. Reduced neural connectivity but increased task-related activity during working memory in de novo Parkinson patients. Hum Brain Mapp. 2015;36(4):1554-66.

27. Simioni AC, Dagher A, Fellows LK. Effects of levodopa on corticostriatal circuits supporting working memory in Parkinson's disease. Cortex. 2017;93:193-205.

\section{Figures}




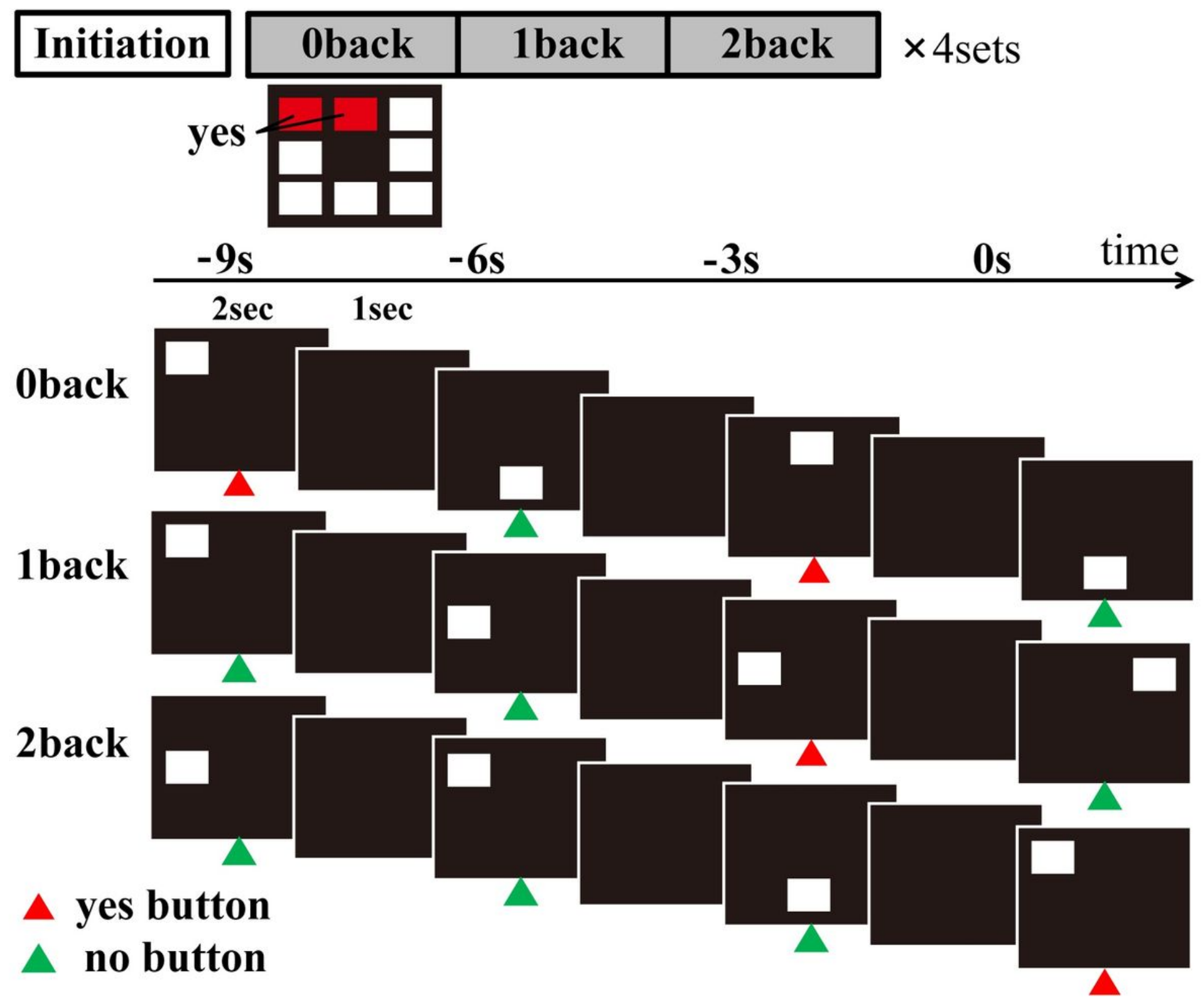

Figure 1

Experimental protocol 
A $\%$

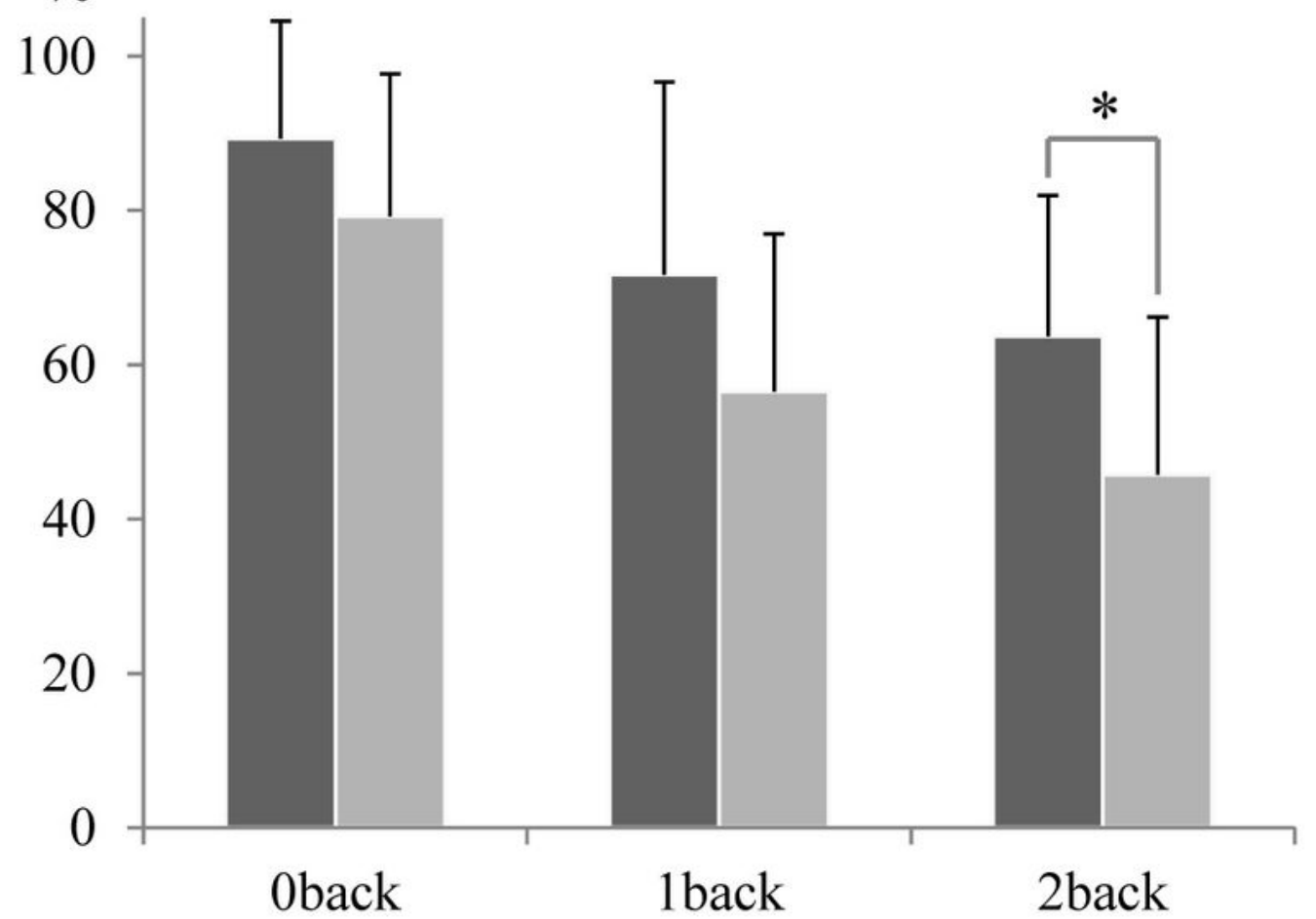

- PDCN

- PDMCI

B msec.

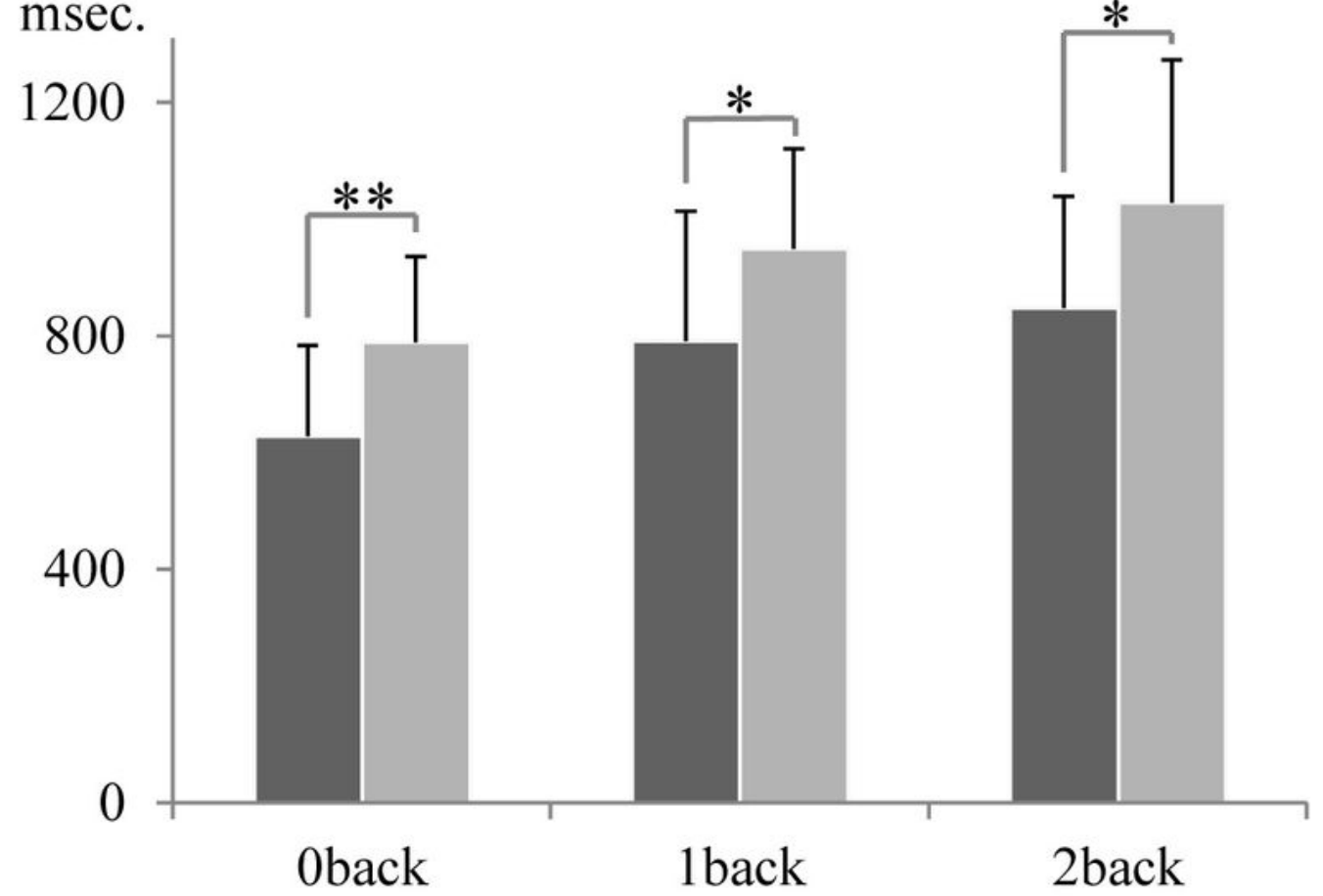

PDCN

PDMCI

\section{Figure 2}

Performance of the visuospatial $n$-back test. A. The figure shows rates of correct answers for the patients with PD-CN and PD-MCl. The scores on the 0 -back test did not differ significantly between groups. The scores on the 2-back test of PD-MCl were significantly lower than that of PD-CN. B. The figure shows the response time to press correct button. The patients with $\mathrm{PD}-\mathrm{MCl}$ took significantly longer time to respond. *P<0.05, ** $P<0.01$. 
A $\%$

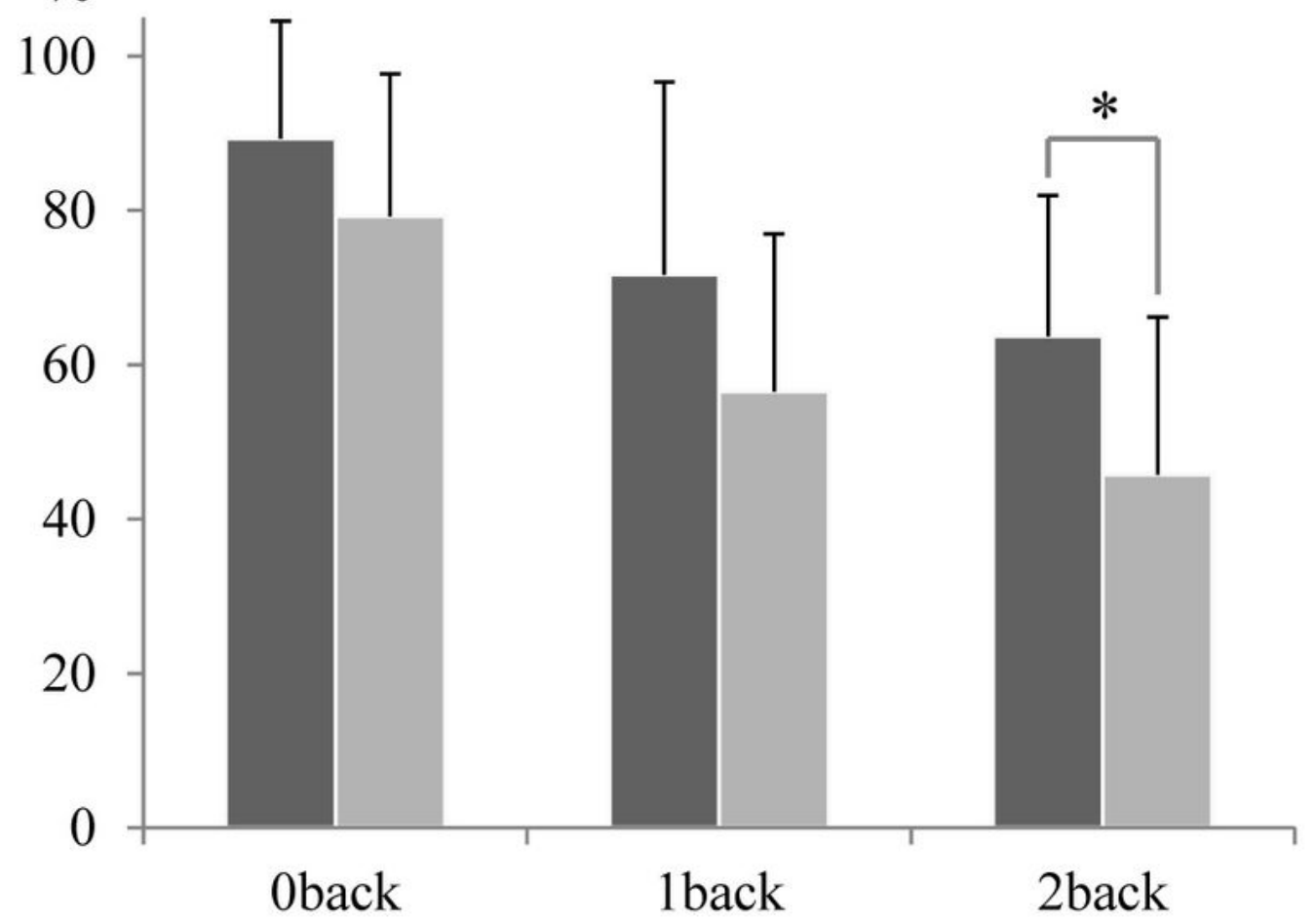

- PDCN

- PDMCI

B msec.

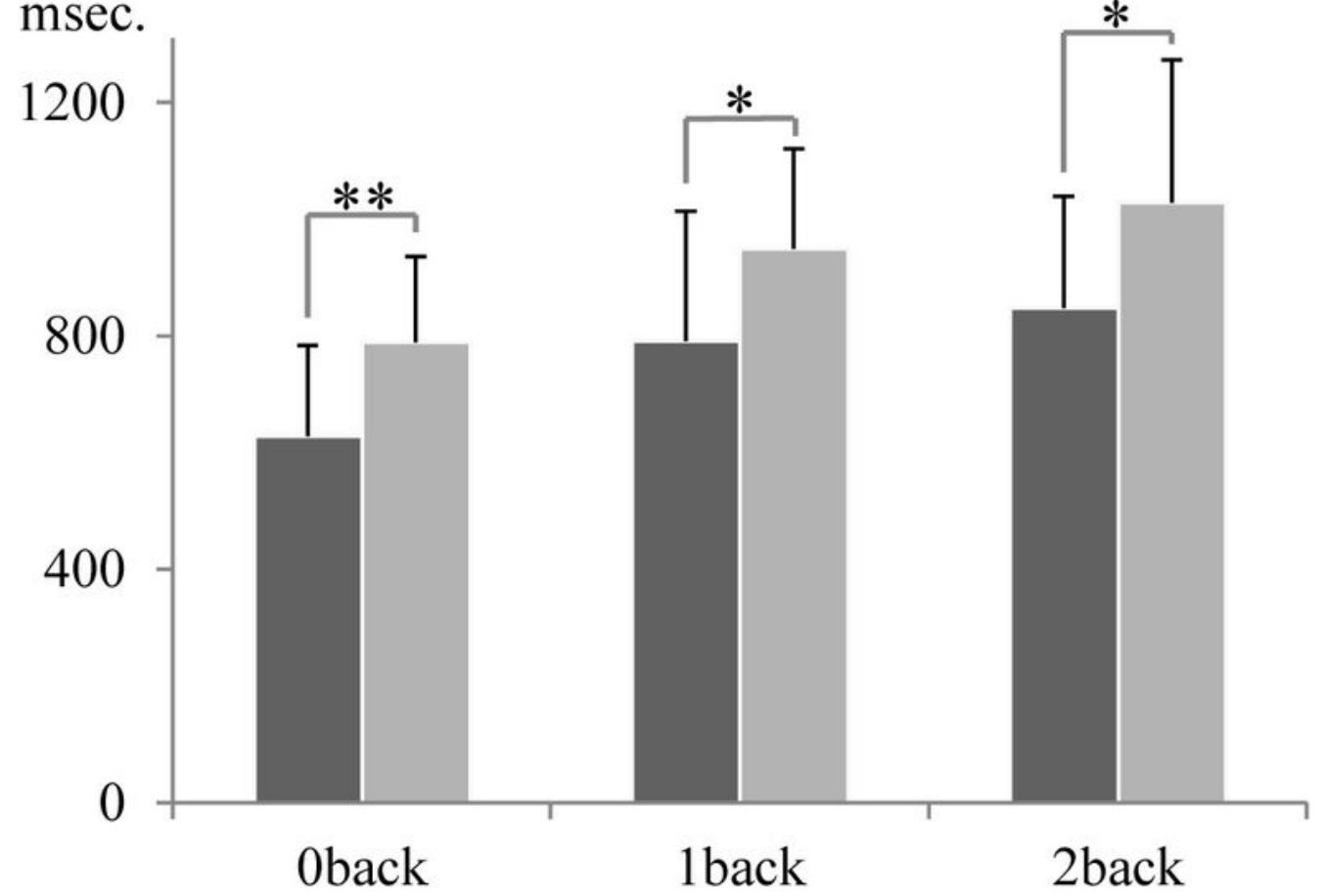

PDCN

PDMCI

\section{Figure 2}

Performance of the visuospatial $n$-back test. A. The figure shows rates of correct answers for the patients with PD-CN and PD-MCl. The scores on the 0 -back test did not differ significantly between groups. The scores on the 2-back test of PD-MCl were significantly lower than that of PD-CN. B. The figure shows the response time to press correct button. The patients with $\mathrm{PD}-\mathrm{MCl}$ took significantly longer time to respond. *P<0.05, ** $P<0.01$. 

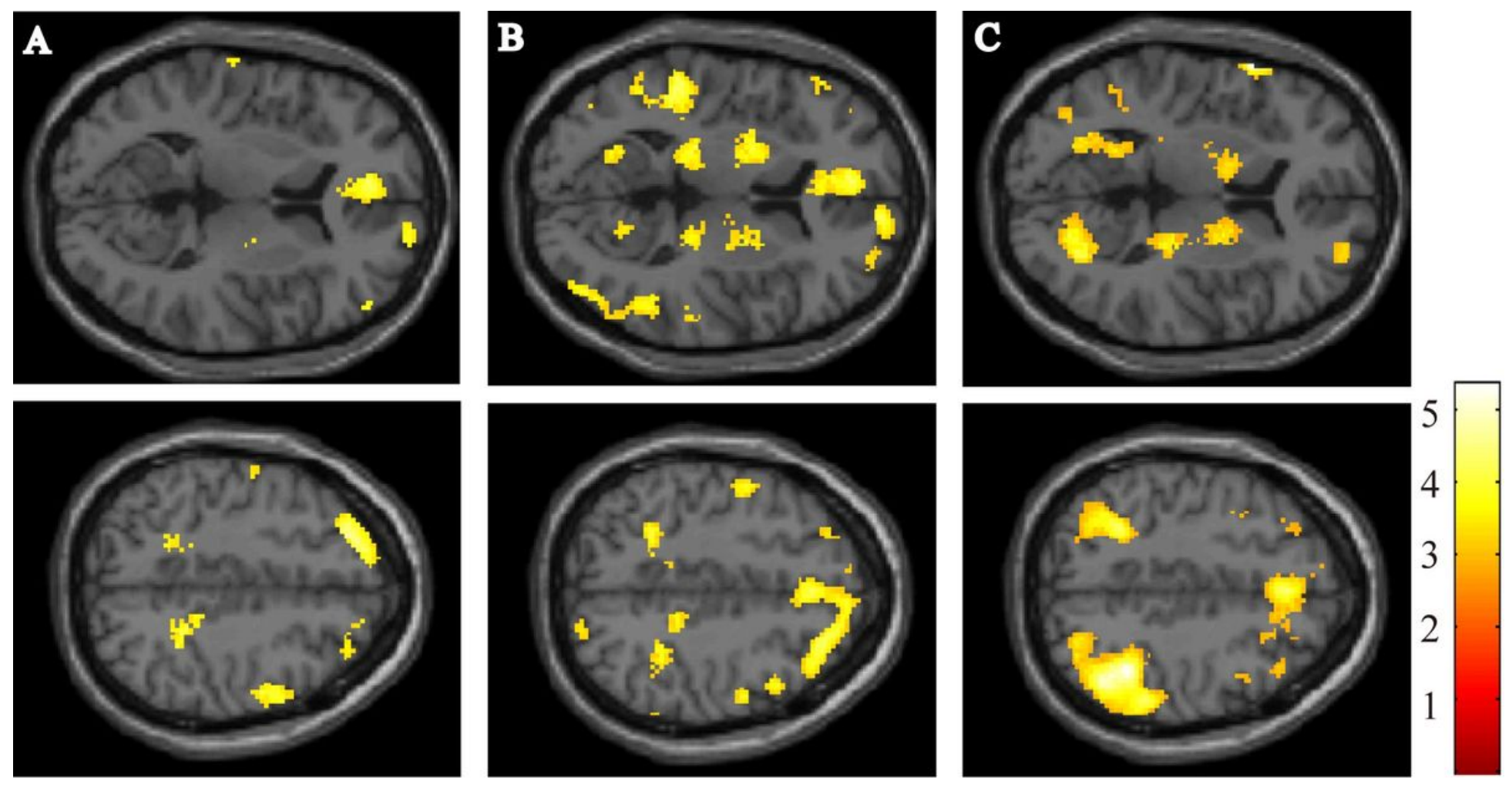

Figure 3

fMRI analyses comparing PD-MCl and PD-CN.
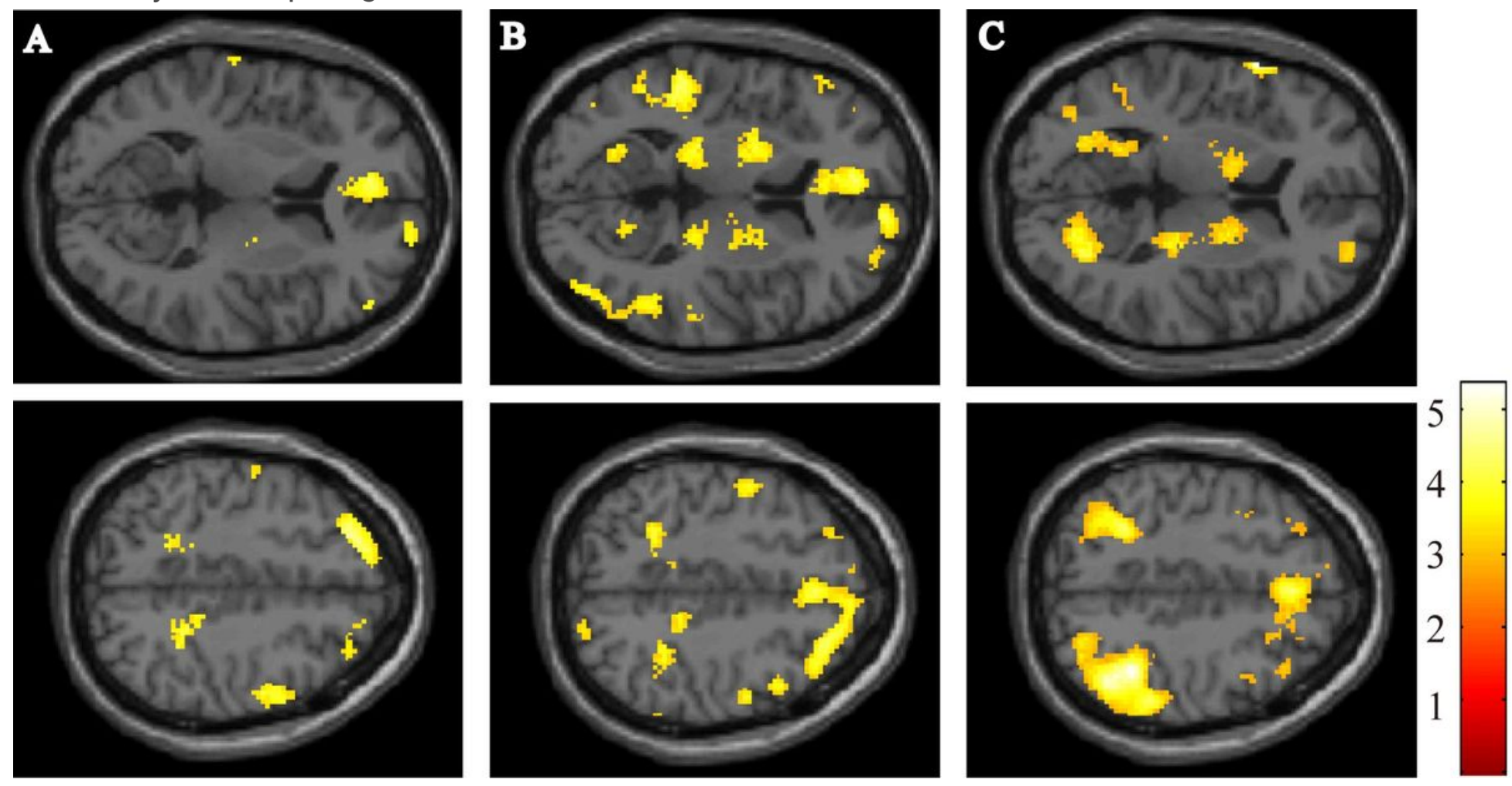

Figure 3

fMRI analyses comparing PD-MCl and PD-CN. 

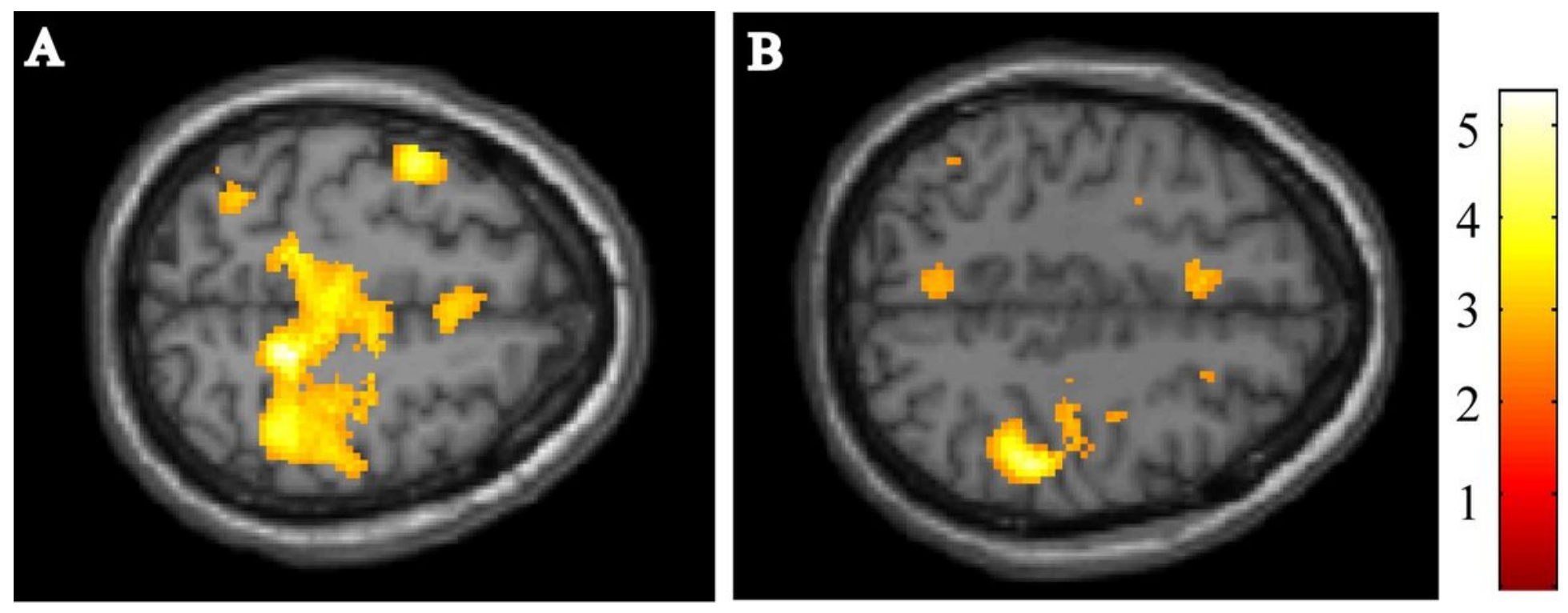

Figure 4

Correlation analyses. A. The image shows the correlation between the scores on the 1-back test and activation of the 1-back test. B. The image shows the correlation between the scores on the 2-back test and activation of the 2-back test.
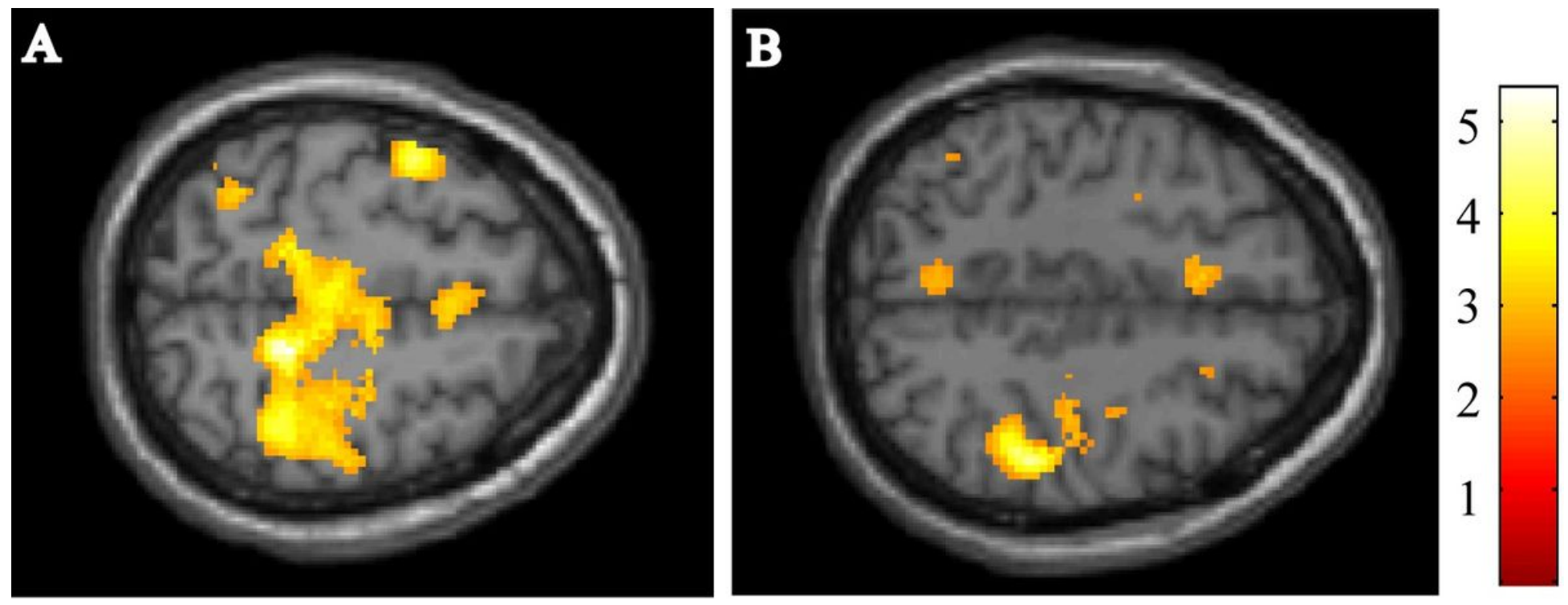

\section{Figure 4}

Correlation analyses. A. The image shows the correlation between the scores on the 1-back test and activation of the 1-back test. B. The image shows the correlation between the scores on the 2-back test and activation of the 2-back test. 